\title{
Portación nasal de Staphylococcus aureus en población pediátrica con epistaxis anterior recurrente
}

\author{
Nasal carriage of Staphylococcus aureus in pediatric population \\ with recurrent anterior epistaxis
}

\author{
Cecilia Sedano $\mathbf{M}^{1}$, Andrés Alvo $\mathbf{V}^{1}$, Daniel Muñoz $\mathbf{S}^{1}$, Daniel Cantero $\mathbf{C}^{1,2}$.
}

\begin{abstract}
RESUMEN
Introducción: La epistaxis es un motivo frecuente de consulta en otorrinolaringología pediátrica. Diversos factores se han asociado, existiendo tanto causas locales como sistémicas. Ciertos estudios en adultos, han sugerido la asociación de colonización nasal por Staphylococcus aureus con la recurrencia de la epistaxis. Este hallazgo en población pediátrica tendría un potencial rol dentro del tratamiento y sería un posible blanco para profilaxis.

Objetivo: Comparar la prevalencia de colonización nasal por $S$. aureus en pacientes pediátricos con epistaxis recurrente, en el Servicio de Otorrinolaringología del Hospital Barros Luco Trudeau, respecto a pacientes sin este antecedente.

Material y método: Estudio caso-control, definiendo caso como pacientes entre 2 y 15 años que consultaron por cuadros de epistaxis anterior recurrente. Se tomaron cultivos nasales a ambos grupos.

Resultados: Se incluyeron 46 pacientes con epistaxis y 45 controles. La prevalencia de $S$. aureus fue de $39 \%$ en los casos y $37 \%$ en los controles, sin diferencias estadísticamente significativas. Al examen físico de la mucosa nasal, ninguna de las características estudiadas mostró una relación significativa con la presencia de $S$. aureus.

Conclusión: La colonización nasal de $S$. aureus de niños con y sin epistaxis recurrente no varía considerablemente. En este estudio, la presencia de $S$. aureus no se asoció estadísticamente a epistaxis recurrente.
\end{abstract}

Palabras clave: Epistaxis, rinología, colonización, profilaxis.

\begin{abstract}
Introduction: Epistaxis is a frequent reason for consultation in pediatric otolaryngology. Several factors have been associated, both local and systemic. Some studies in adult population, have suggested the association of nasal colonization by Staphylococ-
\end{abstract}

\footnotetext{
1 Departamento Otorrinolaringología, Universidad de Chile, Santiago, Chile.

2 Servicio de Otorrinolaringología, Hospital Barros Luco Trudeau, Santiago, Chile.

Los autores declaran no tener conflictos de interés.
}

Recibido el 24 de marzo, 2019. Aceptado el 31 de mayo, 2019. 
cus aureus with the recurrence of epistaxis. Finding this association in pediatric population could have a potential role in treatment and also as a possible target for prophylaxis.

Aim: To compare the prevalence of nasal colonization by $S$. aureus between pediatric patients with recurrent epistaxis and controls with no previous recurrent epistaxis, in the Service of Otorhinolaryngology in Barros Luco Trudeau Hospital.

Material and method: Case-control study, defining case as patients between 2 and 15 years who consulted for recurrent anterior epistaxis. Nasal cultures were taken from both groups.

Results: 46 patients with epistaxis and 45 controls were included. The prevalence of $S$. aureus was $39 \%$ in the case group and $37 \%$ in the controls, without statistically significant differences. At the physical examination of the nasal mucosa, none of the characteristics studied showed a significant relationship with the presence of $S$. aureus.

Conclusion: Nasal colonization of $S$. aureus in children with and without recurrent epistaxis does not vary considerably. In this study, the presence of $S$. aureus was not statistically associated with recurrent epistaxis.

Key words: Epistaxis, rhinology, colonization, prophylaxis.

\section{INTRODUCCIÓN}

La epistaxis es un motivo frecuente de consulta en otorrinolaringología pediátrica'. Localmente, en el Servicio de Otorrinolaringología del Hospital Barros Luco Trudeau, se realizan más de 800 cauterizaciones nasales por epistaxis anterior al año, considerando tanto adultos como niños. Siendo esta una patología prevalente, parece importante conocer factores que podrían favorecer su aparición para así poder prevenirla. La epistaxis recurrente podría llegar a ser potencialmente grave, posee costos asociados a atención en salud y produce disminución de la calidad de vida de los pacientes y sus familias². Diversos factores se han asociado a epistaxis recurrente, que van desde condiciones banales hasta causas neoplásicas de mal pronóstico. Afortunadamente, la gran mayoría corresponden a causas locales benignas, que requieren un manejo conservador. Existen tanto causas locales como sistémicas. Mientras que las causas sistémicas obedecen a trastornos de la coagulación y vasculares, dentro de las locales destaca el grataje digital, patología inflamatoria, trauma nasal, defectos anatómicos y causas tumorales ${ }^{3}$.

Existen publicaciones que han sugerido la asociación de colonización por Staphylococcus aureus (S. aureus) con la recurrencia de la epistaxis.
Ulusoy y cols. demostraron una frecuencia significativamente mayor de $S$. aureus en pacientes con epistaxis, aunque no fueron capaces de distinguir si esto era la causa del sangrado, o consecuencia de la alteración del medio nasal por la presencia de san$\mathrm{gre}^{4}$. En otro estudio, Saafan y cols no lograron demostrar un rol de los biofilms en la epistaxis, aunque sí identificaron cierto grado de inflamación asociada y una mayor prevalencia de bacterias, destacando $S$. aureus ${ }^{5}$. Whymark y cols realizaron un estudio en 2008 en el que encontraron una mayor colonización por $S$. aureus en pacientes con epistaxis respecto a los controles, planteando que podría deberse a neoangiogénesis producto de la inflamación que éste produciría ${ }^{6}$. Por otra parte, Walker y Baring, no lograron demostrar diferencias en el porcentaje de portación nasal de $S$. aureus entre pacientes adultos con epistaxis y el grupo control?.

La posible relación entre la presencia de bacterias y epistaxis no es sólo importante por el potencial rol dentro del tratamiento, sino que también como posible blanco para la profilaxis en casos recurrentes. Existe una publicación en nuestro medio nacional donde se vio que en población adulta con epistaxis recurrente era más frecuente la presencia de $S$. aureus nasal; ; aunque no existen estudios nacionales que evalúen esta asociación en población pediátrica, grupo etario afectado frecuentemente por esta condición. 


\section{OBJETIVO}

La intención de este trabajo es demostrar que existe mayor prevalencia de colonización por $S$. aureus en el vestíbulo nasal de pacientes pediátricos con epistaxis recurrente, consultantes en el Servicio de Otorrinolaringología en el Hospital Barros Luco Trudeau durante los años 2016 y 2017, en comparación con pacientes de las mismas características sin antecedentes de epistaxis previa. Como objetivos secundarios describiremos la portación nasal de $S$. aureus y otros patógenos en población pediátrica y su susceptibilidad a antibióticos; las características de la mucosa nasal en pacientes con epistaxis recurrente con presencia de $S$. aureus y evaluar la posible relación de epistaxis recurrente con las estaciones del año.

\section{MATERIAL Y MÉTODO}

Este trabajo corresponde a un estudio casocontrol realizado en el policlínico del Servicio de Otorrinolaringología del Hospital Barros Luco Trudeau entre los años 2016-2017. Se incluyeron pacientes entre 2 y 15 años que consultaron por cuadros de epistaxis anterior recurrente, definida como 20 más episodios al año de epistaxis anterior, y un grupo control de similares características que consultaron por patología adenoamigdalina, sin historia de epistaxis anterior ni posterior. Cada grupo estuvo formado por 45 pacientes, con un $n$ total de 90 pacientes; el tamaño muestral se calculó utilizando el programa estadístico STATA 12.0, estimando una diferencia de portación de 30\% entre ambos grupos, de acuerdo a lo revisado en la literatura, un error alfa de 0,05 y un poder de $80 \%$. Debido a las razones expuestas por Rose y Laan, se utilizó un muestreo de tipo no-pareado9.

Dado que la rinitis alérgica pudiese ser un factor de confusión, se planificó parear la muestra, sin embargo, esto se dio en forma espontánea ya que 15 pacientes con epistaxis recurrente tenían rinitis alérgica y 16 del grupo control, por lo que no fue necesario intervenir. Se excluyeron pacientes con epistaxis posterior, con coagulopatías o vasculopatías, tumores nasosinusales, uso de fármacos que interfieren con la hemostasia, neoplasias hematológicas, cirugías nasosinusales previas, tratamien- to con corticoides nasales en el último mes, uso de antibiótico local o sistémico en el último mes, e infecciones locales activas.

Frente a la consulta por epistaxis recurrente se preguntó a los pacientes y adultos acompañantes si estaban de acuerdo con participar en el estudio y se solicitó asentimiento y consentimiento informado respectivamente. Se llenó una ficha con la fecha, datos personales, frecuencia de epistaxis, presencia de rinitis alérgica y si estaban en tratamiento. Luego se analizó la anatomía con rinoscopía anterior, se descartó patología tumoral, y se describieron las características de la mucosa. Posteriormente se tomó cultivo de secreción de mucosa septal anterior de la fosa nasal afectada con hisopo; las muestran fueron enviadas para procesamiento al laboratorio de microbiología del Hospital Barros Luco Trudeau.

Se realizó el mismo procedimiento para el grupo control, pacientes consultantes por patología adenoamigdalina sin historia de epistaxis recurrente; intentando tomar el cultivo el mismo día que a los niños con epistaxis recurrente para evitar variaciones según época del año. En el caso de pacientes con epistaxis con cultivo positivo para $S$. aureus se ofreció la alternativa de tratamiento antibiótico tópico con mupirocina. En casos de cultivos positivos para $S$. aureus en el grupo control no se indicó tratamiento, por tratarse solo de colonización sin síntomas asociados.

Los datos se analizaron con el software estadístico STATA 12.0, realizándose un análisis exploratorio de datos, describiendo las variables demográficas de la población estudiada, medidas de tendencia central y proporciones. Se utilizó el test Chi-cuadrado, test exacto de Fisher y test $Z$ para proporciones, para la comparación de ambos grupos, con un valor $\mathrm{p}$ significativo menor a 0,05 . El estudio fue aprobado por el Comité Ético Científico del Servicio de Salud Metropolitano Sur, MEMO 420/2015.

\section{RESULTADOS}

Se incluyeron 46 pacientes en el grupo caso y 45 pacientes en el grupo control; no hubo casos de rechazo a participar en el estudio. El grupo control constó de 13 hombres y 32 mujeres, con un 
promedio de edad de 7,1 años. En el grupo caso, con epistaxis recurrente, 28 pacientes eran de sexo masculino, 18 de sexo femenino, con un mínimo de 2 años, un máximo de 15 años y un promedio de edad de 7,8 años (Tabla 1).

La diferencia entre hombres y mujeres en el grupo caso fue estadísticamente significativa con un valor $p=0,02$. En cuanto a los cultivos, para el grupo caso el $32,6 \%$ obtuvo flora comensal (considerada como Staphylococcus epidermidis y Corynebacterium spp), 71,7\% presentaba algún agente patógeno: S. aureus 39\%, Haemophilus influenzae 13\%, Streptococcus pneumoniae 8,6\%, y Moraxella catarrhalis $10,8 \%$. Los cultivos para el grupo control resultaron en $46,6 \%$ con flora comensal, mientras que $57,7 \%$ presentó algún agente patógeno: $S$. aureus $37 \%, \mathrm{H}$. influenzae
$8,8 \%$, y $S$. pneumoniae $11 \%$. Solo resultó estadísticamente significativa la presencia de $M$. catarrhalis en el grupo caso con un valor $p=0,029$, no así la presencia de $S$. aureus, que no mostró diferencias significativas. La prevalencia global de $S$. aureus fue de $38,4 \%$.

En cuanto a la resistencia a antibióticos solo 2 pacientes, 1 de cada grupo, presentaron $S$. aureus resistente a clindamicina y eritromicina; y un paciente de cada grupo con $S$. pneumoniae resistente a eritromicina. Como objetivo secundario evaluamos las características de la mucosa nasal de los pacientes con epistaxis recurrente, 21 pacientes de 46 presentaban eritema, de los cuales 10 eran $S$. aureus positivo; 2 pacientes presentaron sinequias parciales de mucosa, de los cuales 1 era $S$. aureus positivo (Figura 1); 14 presentaban puentes de

Tabla 1. Resumen de resultados

\begin{tabular}{|c|c|c|c|}
\hline & Epistaxis recurrente & Controles & valor $P$ \\
\hline N Total & 46 & 45 & \\
\hline Hombres & 28 & 13 & 0,02 \\
\hline Mujeres & 18 & 32 & \\
\hline Edad promedio & 7,8 & 7,1 & \\
\hline Flora comensal & $15(32,6 \%)$ & $21(46,6 \%)$ & 0,17 \\
\hline S. aureus & $18(39 \%)$ & $17(37 \%)^{\prime}$ & 0,88 \\
\hline H. influenzae & $6(13 \%)$ & $4(8,8 \%)$ & 0,7 \\
\hline S. pneumoniae & $4(8.6 \%)$ & $5(11 \%)$ & 0,7 \\
\hline M. catarrhalis & $5(10.8 \%)$ & 0 & 0,029 \\
\hline Primavera/Verano & 31 & & 0,0001 \\
\hline Otoño/Invierno & 15 & & \\
\hline
\end{tabular}

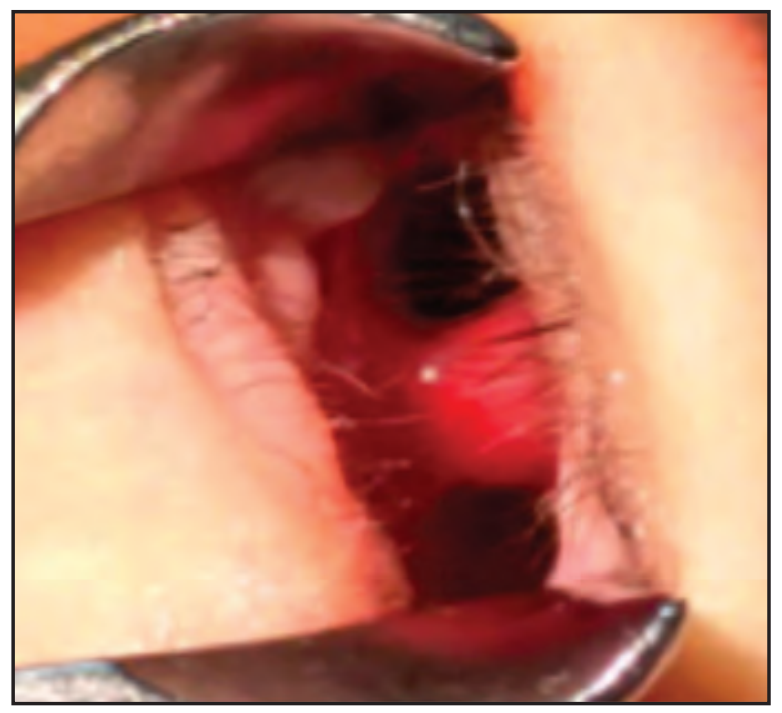

Figura 1. Sinequia parcial de fosa nasal izquierda, luego de cauterizaciones con nitrato de plata a repetición por epistaxis recurrente. 
secreción serosa, 3 de ellos $S$. aureus positivo (Figura 2); costras se observaron en 13 de los 46 pacientes, 8 de ellos $S$. aureus positivo. Ninguna de las características de la mucosa fue estadísticamente significativa para la presencia de $S$. aureus.

Al analizar las estaciones del año en que se presentaron los pacientes con epistaxis recurrente, 12 fueron en verano, 6 en otoño, 9 en invierno y 19 en primavera, no hubo una estación del año estadísticamente distinta a las demás; sin embargo, al analizar el verano en conjunto con la primavera en contraste con otoño-invierno, la diferencia es estadísticamente significativa con un valor $p=$ 0,00001, como suele observarse en la práctica clínica (Figura 3).

\section{DISCUSIÓN}

Como se mencionó en un comienzo, múltiples estudios en población adulta han mostrado mayor frecuencia de $S$. aureus en pacientes con epistaxis recurrente. Sin embargo, en este estudio no hubo una asociación ni tampoco una tendencia, las frecuencias fueron muy similares en ambos grupos. Tampoco se encontró que $S$. aureus pudiese mostrar algún patrón en las características de la mucosa nasal. Es importante mencionar que el porcentaje de portación de $S$. aureus en la población pediátrica de nuestro hospital fue de $38,4 \%$, cifra que deber tenerse en consideración para la indicación de antibióticos en los pacientes con taponamientos nasales, y así prevenir un eventual síndrome de shock tóxico. Es importante mencionar algunos antibióticos a los que $S$. aureus es generalmente sensible y, por ende, se podrían considerar frente a un taponamiento nasal. Tanto las penicilinas resistentes a penicilinasas (cloxacilina, flucloxacilina) como las cefalosporinas de primera generación (cefadroxilo) serían igual de efectivas en el tratamiento de infecciones de tejidos blandos, siendo estas últimas potencialmente menos tóxicas. Por otra parte, la resistencia a amoxicilina es muy alta, lo que puede solucionarse con la adición de un inhibidor de beta-lactamasa (ej.: ácido clavulánico), aunque el espectro antibiótico podría ser más amplio del necesario en este contexto ${ }^{10}$.

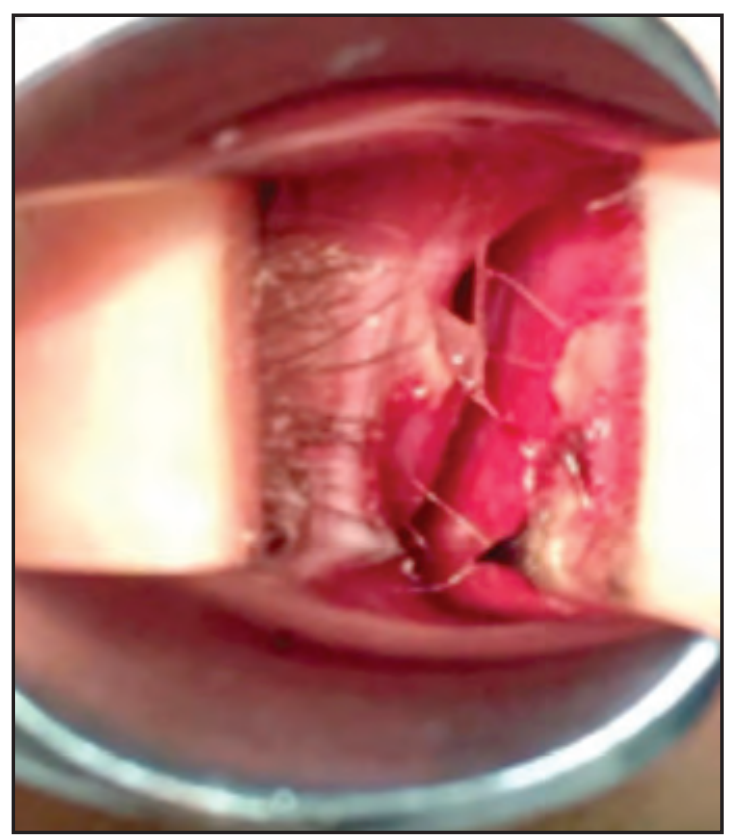

Figura 2. Puentes de secreción mucosa, eritema y costras en fosa nasal derecha. 


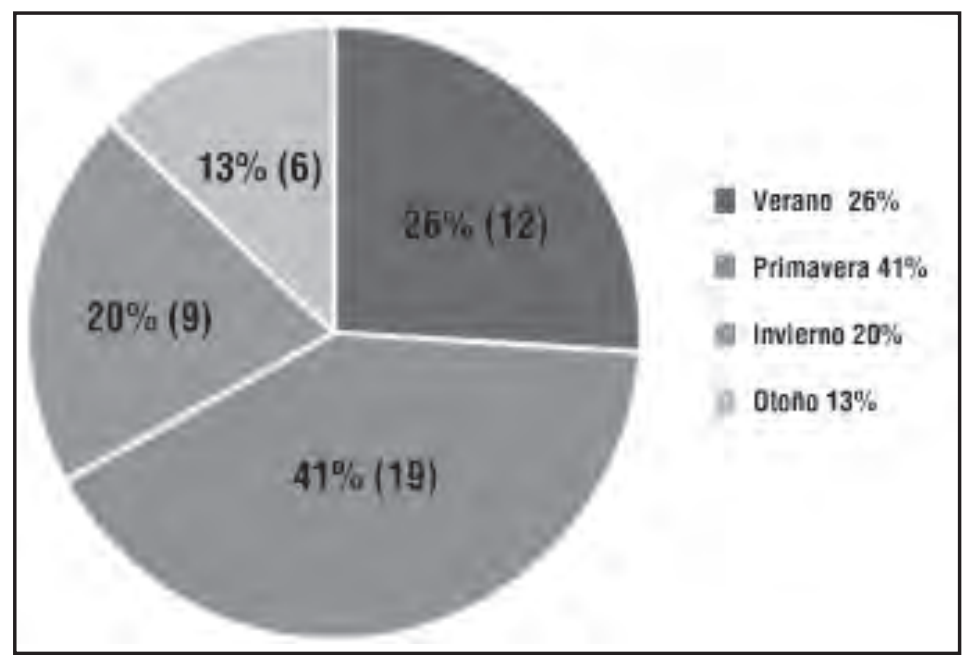

Figura 3. Porcentaje de pacientes con epistaxis recurrente según estación del año.

En caso de alergia a la penicilina, existe $3 \%-$ $8 \%$ de reacción cruzada con cefalosporinas. Por lo tanto, en caso de hipersensibilidad inmediata (urticaria, angioedema, broncoespasmo, anafilaxis) ambos grupos estarían contraindicados; aunque si la respuesta alérgica a penicilina es menor, las cefalosporinas sí podrían ser utilizadas. En estos casos pudiese utilizarse clindamicina o macrólidos, aun cuando la resistencia a estos últimos alcanza cerca de $15 \%{ }^{10}$.

Respecto a la relación entre bacterias y epistaxis, el único patógeno con asociación estadísticamente significativa fue $M$. catarrhalis. Sin embargo, creemos que la presencia en solo 5 de Ios 46 pacientes no permite sacar conclusiones respecto a su real relevancia. Evidenciamos que la epistaxis recurrente es más frecuente en el sexo masculino, esto ya había sido descrito en otras publicaciones, principalmente en pacientes adultos $^{11,12}$. Especulamos que en el caso de los niños pudiese deberse al trauma nasal digital frecuente, pero es solo un supuesto. En Chile, a diferencia de lo descrito en países del hemisferio norte ${ }^{12,13}$, es frecuente ver que la consulta por epistaxis aumenta en los meses de primavera y verano. En este estudio, estas estaciones concentraron el mayor número de pacientes con epistaxis recurrente, y planteamos que pudiese deberse a alergenos que aumentan en este periodo, el uso de aire acondicionado u otros irritantes; 0 es posible que las altas temperaturas generen mayor vasodilatación aumentando la frecuencia de epistaxis.

\section{CONCLUSIÓN}

En este trabajo observamos que la flora bacteriana nasal de niños con y sin epistaxis recurrente no varía considerablemente. Todos los agentes bacterianos resultaron altamente sensibles en el antibiograma. La portación de $S$. aureus global en población pediátrica entre 2 y 15 años en nuestro servicio fue de $38,4 \%$ y no se asoció estadísticamente a epistaxis recurrente; por lo que según este estudio no se justificaría el uso de mupirocina ni otros antibióticos tópicos nasales en población pediátrica con epistaxis recurrente. 


\section{BIBLIOGRAFÍA}

1. QUREISHI A, BuRTon MJ. Interventions for recurrent idiopathic epistaxis (nosebleeds) in children. Cochrane Database Syst Rev 2012; 12; 9: CD004461.

2. Davies K, Batra K, Mehanna R2, Keogh I. Pediatric epistaxis: epidemiology, management \& impact on quality of life. Int J Pediatr Otorhinolaryngol 2014; 78: 1294-7.

3. KuBBa H. Childhood epistaxis. Clin Otolaryngol 2006; 31: 212-13.

4. Ulusoy S, Babagglu G, Catli T, San T, Cingi C. Epistaxis and Staphylococcus aureus colonization in the nasal vestibule: is it a cause or consequence? J Craniofac Surg 2014; 25: 513-5.

5. SaAfan ME, IbRahim WS. Role of bacterial biofilms in idiopathic childhood epistaxis. Eur Arch Otorhinolaryngol 2013; 270: 909-14.

6. Whymark AD, Crampsey dP, Fraser L, Moore P, Williams C, Kubba H. Childhood epistaxis and nasal colonization with Staphylococcus aureus. Otolaryngol Head Neck Surg 2008; 138: 307-10.

7. WalkeR FD, BARING DE. Nasal bacterial carriage in adult epistaxis: is neomycin the answer? $J$ Laryngol Otol 2009; 123: 623-5.

8. Bertoglia F, Brunetto B. Algunos aspectos de la bacteriología de las epistaxis. Rev Otorrinolaring 1976; 36: 77-8.

9. RoSE S, LAAN MJ. Why match? Investigating matched case-control study designs with causal effect estimation. Int J Biostat 2009; 5: 1.

10. Rayner C, Munckhof W.J. Antibiotics currently used in the treatment of infections caused by Staphylococcus aureus. Internal Medicine Journal 2005; 35: S3-S16.

11. Côrte FC, Orfao T, Dias CC, Moura CP, Santos M. Risk factors for the occurrence of epistaxis: Prospective study. Auris Nasus Larynx2017; 22: S0385-8146(17)30095-0.

12. Chaaban MR, Zhang D, Resto V, Goodwin JS. Demographic, Seasonal, and Geographic Differences in Emergency Department Visits for Epistaxis. Otolaryngol Head Neck Surg 2017; 156: 81-6.

13. Purkey MR, Seeskin Z, Chandra R. Seasonal variation and predictors of epistaxis. Laryngoscope 2014; 124: 2028-33.

\footnotetext{
Correspondencia: Cecilia Sedano Muñoz Servicio de Otorrinolaringología, Hospital Barros Luco Trudeau

Gran Avenida 3024, San Miguel, Santiago, Chile

E mail: cecisedano@gmail.com
} 